\title{
On the Existence and Uniqueness of Solutions of Boundary Value Problems for Second Order Functional Differential Equations
}

\author{
Evangelia S. AthanAssiadou
}

\begin{abstract}
In this paper we study existence and uniqueness of solutions of boundary value problems for second order nonlinear delay differential equations. We transform the boundary value problem to an equivalent integral equation and then we use the Banach fixed point theorem and the notion of the Fréchet derivative.
\end{abstract}

\section{INTRODUCTION}

This paper is concerning with the existence and uniqueness of solutions of boundary value problems for nonlinear second order functional differential equations. More precisely, we study boundary value problems for the equations of the form

$$
x^{\prime \prime}(t)=f\left(t, x_{t}\right),
$$

where $f$ is a continuous function and $x_{t}$ is a function which denote the delay. The boundary conditions are consisted from the relation $x_{0}=\phi$ or equivalent $x(s)=\varphi(s)$ on an interval as well as a condition which connect the values of $x$ on some points of its domain. In this paper general boundary conditions are considered which cover known cases as in [6] and [8]. Existence and uniqueness are proved in [8] by using the fixed point technique, in [7] the topological degree method is applied, while in [6] the method of quasilinearization has been extended to boundary value problems for functional differential equations of second order. In [10] and [9] boundary value problems for first and second order functional differential equations respectively are studied via the method of upper and lower solutions. Existence and dependence of solutions results for similar problems there exist in [5] and [1]. For the basic theory of functional differential equations we refer to the books [2], [3] and [4].

2010 Mathematics Subject Classification. Primary: 34H05, 43A75.

Key words and phrases. Functional differential equation, integral equation, contractive operator. 
In section 2 we transform the boundary value problem to an equivalent integral equation. In section 3 we construct an contractive operator and applying the Banach fixed point theorem we prove an existence and uniqueness result. We obtain corresponding results using the notion of the Fréchet derivative.

\section{Preliminaries and notations}

For a positive number $\tau$ the space of all continuous functions $\varphi:[-\tau, 0] \rightarrow$ $\mathbb{R}$ will be denoted by $C=C([-\tau, 0], \mathbb{R})$. The supremum norm of $\varphi \in$ $C([-\tau, 0], \mathbb{R})$ is defined by

$$
\|\varphi\|_{C}=\sup \{|\varphi(t)|: t \in[-\tau, 0]\} .
$$

For every continuous function $x:[-\tau, b] \rightarrow \mathbb{R}, b>0$, and every $t \in[0, b]$ we denote by $x_{t}$ the function $x_{t}:[-\tau, 0] \rightarrow \mathbb{R}$ with

$$
x_{t}(s)=x(t+s), s \in[-\tau, 0] .
$$

Especially the condition $x_{0}=\varphi$ is equivalent to $x(s)=\varphi(s), s \in[-\tau, 0]$. This paper is concerned with the boundary value problem

$$
\begin{gathered}
x^{\prime \prime}(t)=f\left(t, x_{t}\right), \quad 0 \leq t \leq b, \\
x_{0}=\varphi, \\
a x(0)+\beta x^{\prime}(0)+\gamma x(b)+\delta x^{\prime}(b)=\lambda,
\end{gathered}
$$

where $f:[0, b] \times C \rightarrow \mathbb{R}$ is a continuous function, $\varphi \in C$, and $a, \beta, \gamma, \delta, \lambda \in \mathbb{R}$ with $\beta+\delta+b \gamma \neq 0$ and $a, \lambda$ arbitrary numbers.

Lemma 1. Boundary value problem (1)-(3) is equivalent to the integral equation

$$
\begin{aligned}
& x(t)=\varphi(0)+\frac{t}{\beta+\delta+\gamma b}\left[\lambda-(\alpha+\gamma) \varphi(0)-\int_{0}^{b}(\delta+\gamma b-\gamma s) f\left(s, x_{s}\right) \mathrm{d} s\right] \\
& \quad+\int_{0}^{t}(t-s) f\left(s, x_{s}\right) \mathrm{d} s, \quad t \in[0, b], \\
& x(t)=\varphi(t), \quad t \in[-\tau, 0] .
\end{aligned}
$$

Proof. Let $x$ be a solution of (1)-(3) then integrating by parts (1) in the interval $[0, t]$ we have

$$
x^{\prime}(t)-x(0)=\int_{0}^{t} f\left(s, x_{s}\right) \mathrm{d} s
$$

and putting $t=b$ we take

$$
x^{\prime}(0)-x^{\prime}(b)=-\int_{0}^{b} f\left(s, x_{s}\right) \mathrm{d} s
$$


Integrating (4) again in $[0, t]$ we have

$$
x(t)-x(0)-t x^{\prime}(0)=\int_{0}^{t} \int_{0}^{\tau} f\left(s, x_{s}\right) \mathrm{d} s \mathrm{~d} \tau
$$

which is becomes

$$
x(t)-t x^{\prime}(0)=\varphi(0)+\int_{0}^{t}(t-s) f\left(s, x_{s}\right) \mathrm{d} s .
$$

From (6) with $t=b$ we take

$$
x(b)-b x^{\prime}(0)=\varphi(0)+\int_{0}^{b}(t-s) f\left(s, x_{s}\right) \mathrm{d} s .
$$

From the linear system (3), (5) and (7) we have

(8) $x^{\prime}(0)=\frac{1}{\beta+\delta+\gamma b}\left[\lambda-(\alpha+\gamma) \varphi(0)-\int_{0}^{b}(\delta+\gamma b-\gamma s) f\left(s, x_{s}\right) \mathrm{d} s\right]$.

From (6) and (8) we have

(9)

$$
\begin{aligned}
x(t)=\varphi & (0)+\frac{t}{\beta+\delta+\gamma b}\left[\lambda-(\alpha+\gamma) \varphi(0)-\int_{0}^{b}(\delta+\gamma b-\gamma s) f\left(s, x_{s}\right) \mathrm{d} s\right] \\
& +\int_{0}^{t}(t-s) f\left(s, x_{s}\right) \mathrm{d} s .
\end{aligned}
$$

Conversely, by differentiating (9) we take

$$
\begin{aligned}
x^{\prime}(t)= & \frac{1}{\beta+\delta+\gamma b}\left[\lambda-(\alpha+\gamma) \varphi(0)-\int_{0}^{b}(\delta+\gamma b-\gamma s) f\left(s, x_{s}\right) \mathrm{d} s\right] \\
& \quad+\int_{0}^{t} f\left(s, x_{s}\right) \mathrm{d} s, \\
x^{\prime \prime}(t)= & f\left(t, x_{t}\right), t \in[0, b] .
\end{aligned}
$$

Taking into account (9) and (10) we calculate to (3).

\section{MAIN RESULT}

In this section we present an existence and uniqueness result for the boundary value problem (1)-(3).

Theorem 1. Let $f:[0, b] \times C \rightarrow \mathbb{R}$ be a continuous function. We assume that there exists an integrable and positive function $m$ such that

$$
|f(t, \psi)-f(t, \bar{\psi})| \leq m(t)\|\psi-\bar{\psi}\|_{C},
$$

for $t \in[0, b], \psi, \bar{\psi} \in C$ and

$$
\ln \left(1+\frac{|\beta+\delta+\gamma b|}{A}\right)>b M(b),
$$


where $M(t)=\int_{0}^{t} m(\tau) \mathrm{d} \tau$ and $A=\max _{s \in[0, b]}|\delta+\gamma b-\gamma s|>0$. Then, the problem (1)-(3) has a unique solution.

Proof. For $x \in C$ we define the norm

$$
\|x\|=\max \left\{e^{-\rho M(t)} \max \{|x(s)|, s \in[0, t]\}, t \in[0, b]\right\},
$$

where

$$
b<\rho<\frac{1}{M(b)} \ln \left(1+\frac{|\beta+\delta+\gamma b|}{A}\right) .
$$

Also we define the operator $P: C \rightarrow C$, by

$$
\begin{gathered}
(P x)(t)=\varphi(0)+\frac{t}{\beta+\delta+\gamma b}\left[\lambda-(\alpha+\gamma) \varphi(0)-\int_{0}^{b}(\delta+\gamma b-\gamma s) f\left(s, x_{s}\right) \mathrm{d} s\right] \\
+\int_{0}^{t}(t-s) f\left(s, x_{s}\right) \mathrm{d} s, \quad t \in[0, b] .
\end{gathered}
$$

Taking into account (11), for any $x, y \in C$ we have

$$
\begin{aligned}
|(P x)(t)-(P y)(t)| \leq \mid & \frac{t}{\beta+\delta+\gamma b}\left|\int_{0}^{b}\right| \delta+\gamma b-\gamma s|\cdot| f\left(s, x_{s}\right)-f\left(s, y_{s}\right) \mid \mathrm{d} s \\
& \quad+\int_{0}^{t}(t-s)\left|f\left(s, x_{s}\right)-f\left(s, y_{s}\right)\right| \mathrm{d} s \\
\leq & \frac{A b}{|\beta+\delta+\gamma b|} \int_{0}^{b} m(s)\left\|x_{s}-y_{s}\right\|_{C} \mathrm{~d} s \\
& +b \int_{0}^{t} m(s)\left\|x_{s}-y_{s}\right\|_{C} \mathrm{~d} s .
\end{aligned}
$$

For $s \in[0, \tau]$ we have

$$
\begin{aligned}
\left\|x_{s}-y_{s}\right\|_{C} & =\sup _{r \in[-\tau, 0]}|x(s+r)-y(s+r)| \\
& =\max _{r \in[-s, 0]}|x(s+r)-y(s+r)| \\
& =\max _{r \in[0, s]}|x(r)-y(r)| .
\end{aligned}
$$

Similarly for $s \in(\tau, b]$ we have

$$
\begin{aligned}
\left\|x_{s}-y_{s}\right\| & \leq \sup _{r \in[-\tau, 0]}|x(s+r)-y(s+r)| \\
& =\max _{r \in[s-\tau, s]}|x(s+r)-y(s+r)| \\
& \leq \max _{r \in[0, s]}|x(r)-y(r)| .
\end{aligned}
$$


Therefore

$$
\begin{aligned}
& |(P x)(t)-(P y)(t)| \\
\leq & \frac{A b}{|\beta+\delta+\gamma b|} \int_{0}^{b} m(s) e^{\rho M(s)} e^{-\rho M(s)} \max _{r \in[0, s]}|x(r)-y(r)| \mathrm{d} s \\
& \quad+b \int_{0}^{t} m(s) e^{\rho M(s)} e^{-\rho M(s)} \max _{r \in[0, s]}|x(r)-y(r)| \mathrm{d} s \\
\leq & \frac{A b}{|\beta+\delta+\gamma b|}\|x-y\| \int_{0}^{b} m(s) e^{\rho M(s)} \mathrm{d} s+b\|x-y\| \int_{0}^{t} m(s) e^{\rho M(s)} \mathrm{d} s \\
= & \frac{A b}{|\beta+\delta+\gamma b|}\|x-y\| \frac{e^{\rho M(b)}-1}{\rho}+b\|x-y\| \frac{e^{\rho M(t)}-1}{\rho} \\
= & \frac{b}{\rho}\|x-y\|\left[\frac{A}{|\beta+\delta+\gamma b|}\left(e^{\rho M(b)}-1\right)+e^{\rho M(t)}-1\right] \\
= & \frac{b}{\rho}\|x-y\|\left[\frac{1}{|\beta+\delta+\gamma b|}\left(A e^{\rho M(b)}-A-|\beta+\delta+b \gamma|\right)+e^{\varrho M(t)}\right]
\end{aligned}
$$

From (12) it follows

$$
A e^{\rho M(b)}-A-|\beta+\delta+b \gamma|<0 .
$$

Hence we have

$$
|(P x)(t)-(P y)(t)| \leq \frac{b}{\rho}\|x-y\| e^{\rho M(t)} .
$$

From (15) it follows

$$
\max _{s \in[0, t]}|(P x)(s)-(P y)(s)| \leq \frac{b}{\rho}\|x-y\| \max _{s \in[0, t]} e^{\rho M(s)} \leq \frac{b}{\rho}\|x-y\| e^{\rho M(t)},
$$

and hence

$$
\max _{t \in[0, b]}\left[e^{-\rho M(t)} \max _{s \in[0, t]}|(P x)(s)-(P y)(s)|\right] \leq \frac{b}{\rho}\|x-y\| .
$$

Therefore

$$
\left\|P_{x}-P_{y}\right\| \leq \frac{b}{\rho}\|x-y\| .
$$

From (16), where $\frac{b}{\rho}<1$, we conclude that $P$ is an contractive operator and applying the Banach fixed point theorem, $P$ has a unique fixed point in $C$ and the theorem is proved.

Theorem 2. Let $f:[0, b] \times C \rightarrow \mathbb{R}$ be a continuous function. We assume that the Fréchet derivative $f_{\Phi}$ exists, is continuous and satisfies

$$
\left|f_{\Phi}(t, \Phi) u\right| \leq K\|u\|_{C}
$$


for $t \in[0, b], \Phi, u \in C$ and

$$
0 \leq K<\frac{1}{b^{2}} \ln \left(1+\frac{|\beta+\delta+\gamma b|}{A}\right)
$$

Then the problem (1)-(3) has a unique solution.

Proof. Taking into account (17) we conclude that (11) is satisfied with $m(t)=K$. Also we have $M(t)=K t$. From (18) we take

$$
0 \leq K b<\frac{1}{b} \ln \left(1+\frac{|\beta+\delta+\gamma b|}{A}\right)
$$

that is the condition (12) is valid. In accordance to Theorem 1 the problem (1)-(3) has a unique solution.

Example 1. Consider the boundary value problem

$$
\begin{gathered}
x^{\prime \prime}(t)=e^{t}+\frac{1}{8}(\sin t) \int_{-2}^{0} x_{t}(s) \mathrm{d} s, \quad t \in[0,1], \\
x(s)=(s+1)^{2}, \quad s \in[-2,0], \\
4 x(0)+2 x^{\prime}(0)+x(1)-x^{\prime}(1)=5 .
\end{gathered}
$$

Here we have $a=4, \beta=2, \gamma=1, \delta=-1, \lambda=5, b=1$ and

$$
\mid f(t, u)-f(t, \bar{u}) \leq \frac{1}{4}\|u-\bar{u}\|_{C}
$$

for $t \in[0,1]$ and $u, \bar{u} \in C$. Then for $m(t)=\frac{1}{4}$ we have $M(t)=\frac{1}{4} t$ and $M(1)=\frac{1}{4}$. Also, it is $A=\max _{s \in[0,1]}|-1+1-s|=1$ and the assumption (12) is satisfied. Hence the boundary value problem (19)-(21) has a unique solution.

\section{REFERENCES}

[1] E. Athanasiadou, On the continuous dependence of solutions of boundary value problems for delay differential equations, in press, 2013.

[2] O. Diekmann, S. A. Van Gils, S. M. Verdyn Lunel and H. O. Walther, Delay Equations:Functional-Compolex and Nonlinear Analysis, New York, 1995.

[3] R. D. Driver, Ordinary and Delay Differential Equations, Springer-Verlag New York, 1977.

[4] J. K. Hale, Functional differential, Vol. 3, Springer-Verlag, 1971.

[5] M. Jablonski and T. Twardowska, On Boundary Value problems for differential equations with a retarded argument, U. I. acta Math. XXVI, 29-36.

[6] T. Jankowshi, Functional differential equations of seconf order, Bull. Belg. Math. Soc. 10 (2003), 291-298.

[7] W. Peixuan, Boundary value problems for second order mixed-type functional differential equations, Appl. Math. JCU, 12B (1997), 155-164. 
[8] L. Skóra, Boundary value problems for second order delay differential equations, Opuscula Mathematica, Vol. 32, No. 3 (2012), 551-558.

[9] W. B. Wang, J. H. Shen, Z. G. Luo, Multi-point boundary value problems for secondorder functional differential equations, J. Comput. Math. Appl. 56 (2008), 2065-2072.

[10] S. Wu-Jun, Nonlinear boundary value problems for discontinuous delayed differential equations, Appl. Math. JCU, 25(1) (2010), 9-17.

Evangelia S. Athanassiadou

Department of Mathematics

University OF ATHENS

GR-15784, Panepistimiopolis Athens

Greece

E-mail address: eathan@math.uoa.gr 